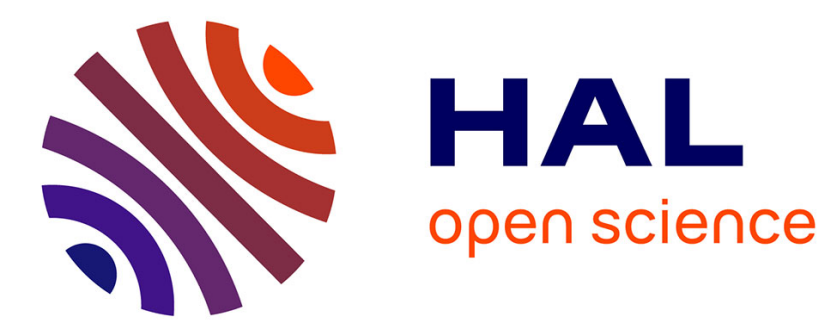

\title{
Accompagner la transition vers des mobilités plus durables dans le périurbain
}

\author{
Anne Aguilera, Benoit Conti, Florent Le Néchet
}

\section{To cite this version:}

Anne Aguilera, Benoit Conti, Florent Le Néchet. Accompagner la transition vers des mobilités plus durables dans le périurbain. Transports urbains: mobilité, réseaux, territoires, 2017, 130. halshs01527428

\section{HAL Id: halshs-01527428 \\ https://shs.hal.science/halshs-01527428}

Submitted on 24 May 2017

HAL is a multi-disciplinary open access archive for the deposit and dissemination of scientific research documents, whether they are published or not. The documents may come from teaching and research institutions in France or abroad, or from public or private research centers.
L'archive ouverte pluridisciplinaire HAL, est destinée au dépôt et à la diffusion de documents scientifiques de niveau recherche, publiés ou non, émanant des établissements d'enseignement et de recherche français ou étrangers, des laboratoires publics ou privés. 


\title{
Accompagner la transition vers des mobilités plus durables dans le périurbain
}

\author{
Anne Aguiléra, Benoit Conti, Florent Le Néchet
}

Aguilera, A., Conti, B., \& Le Néchet, F. (2017). Accompagner la transition vers des mobilités plus durables dans le périurbain. Transports Urbains-mobilités réseaux territoires, $\mathrm{N}^{\circ} 130$, 3-9

Régulièrement critiqué (Billard et Brennetot, 2009) au nom d'arguments financiers (coûts du raccordement aux réseaux de transport et d'énergie), environnementaux (consommation de terres agricoles, émission de gaz à effet de serre en lien avec la dépendance automobile ${ }^{1}$ ), esthétiques ("laideur » des zones pavillonnaires), ou encore socio-politiques (logiques individualistes et recherche de l'entre-soi), le processus de périurbanisation bénéficie progressivement d'un diagnostic plus équilibré (Bailly et Bourdeau-Lepage, 2011 ; Charmes, 2007 ; Vanier et Roux, 2008). Ce, au regard d'avantages qui concernent ses ressources agricoles (permettant d'envisager la mise en place de circuits courts pour alimenter les métropoles), la fourniture de services éco-systémiques (biomasse, séquestration de carbone, paysages) ou encore la redynamisation de petites communes autrefois dépeuplées par l'exode rural. Cette lente "réhabilitation » de la périurbanisation et de ses habitants procède, également, du constat d'un renouvellement des formes du peuplement et des modes de vie qui battent en brèche un certain nombre des critiques ayant alimenté le diagnostic d'incompatibilité avec les trois piliers du développement durable (Feildel et al., 2014).

Dans la lignée des travaux qui cherchent à renouveler le regard sur les liens entre périurbanisation et

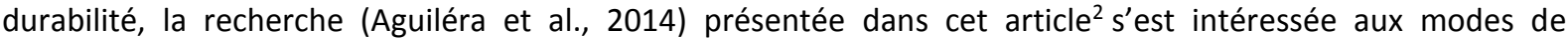
peuplement et aux mobilités quotidiennes des habitants de trois territoires situés dans le périurbain francilien (Figure 1). Ces derniers ont en commun d'être à une distance quasi-égale de Paris (une trentaine de kilomètres, environ), d'avoir été tenus à l'écart de projets majeurs d'aménagement urbain ou de transport, et de former des EPCI (Etablissement Public de Coopération Intercommunale) : ces deux dernières précautions pour nous assurer, d'une part, que la dynamique de périurbanisation avait gagné nos territoires indépendamment d'une volonté politique régionale ou nationale, et d'autre part, pour constituer des territoires ayant une unité institutionnelle et un avenir pensés sur une base collective.

Cette recherche a mobilisé des matériaux de nature variée : des données de recensement (RP) et d'enquête de transport (Enquête Globale de Transport, ou EGT), des réponses à un questionnaire original distribué dans plusieurs communes localisées sur notre terrain d'étude et rempli par plus de 300 ménages (constituant un échantillon représentatif de la population de nos communes d'étude), ainsi que des entretiens réalisés auprès d'une trentaine de résidents. Ces deux enquêtes (par questionnaire et par entretien) visaient la compréhension des logiques de l'implantation périurbaine et des modes de vie (territoires fréquentés et mobilités) des habitants, ainsi qu'une exploration de leurs marges de manœuvre pour diminuer leur dépendance à la voiture, notamment dans un scénario d'augmentation forte du coût des carburants (d'autant plus plausible qu'une hausse significative avait eu lieu quelques mois auparavant).

Si nos analyses concernent trois territoires spécifiques, les fortes ressemblances que l'on observe ainsi que la grande convergence avec d'autres terrains, notamment franciliens (Berger et al., 2014), suggèrent que

\footnotetext{
${ }^{1}$ En moyenne, un périurbain émet trois fois plus de $\mathrm{CO}^{2}$ qu'un habitant du centre et de sa proche banlieue pour se rendre sur son lieu de travail ou d'études car il utilise plus la voiture et parcourt une distance moyenne presque trois fois plus élevée. (Insee Première $N^{\circ} 1357$ - juin 2011)

${ }^{2}$ Recherche financée par le PUCA (Plan Urbanisme Construction Architecture).
} 
beaucoup de nos résultats ont une portée assez générale et rendent finalement compte d'un certain nombre d'invariants de la périurbanisation autour des grandes villes françaises, qui nous permettent de dessiner de nouvelles pistes pour les territoires périurbains au regard des impératifs de la mobilité durable. Après avoir dressé un bilan de l'évolution des mobilités, résidentielle et quotidienne, des perspectives de réflexion d'évolution de l'action publique seront proposées en lien avec les principaux résultats des différentes enquêtes.

Figure 1 : Les trois territoires étudiés

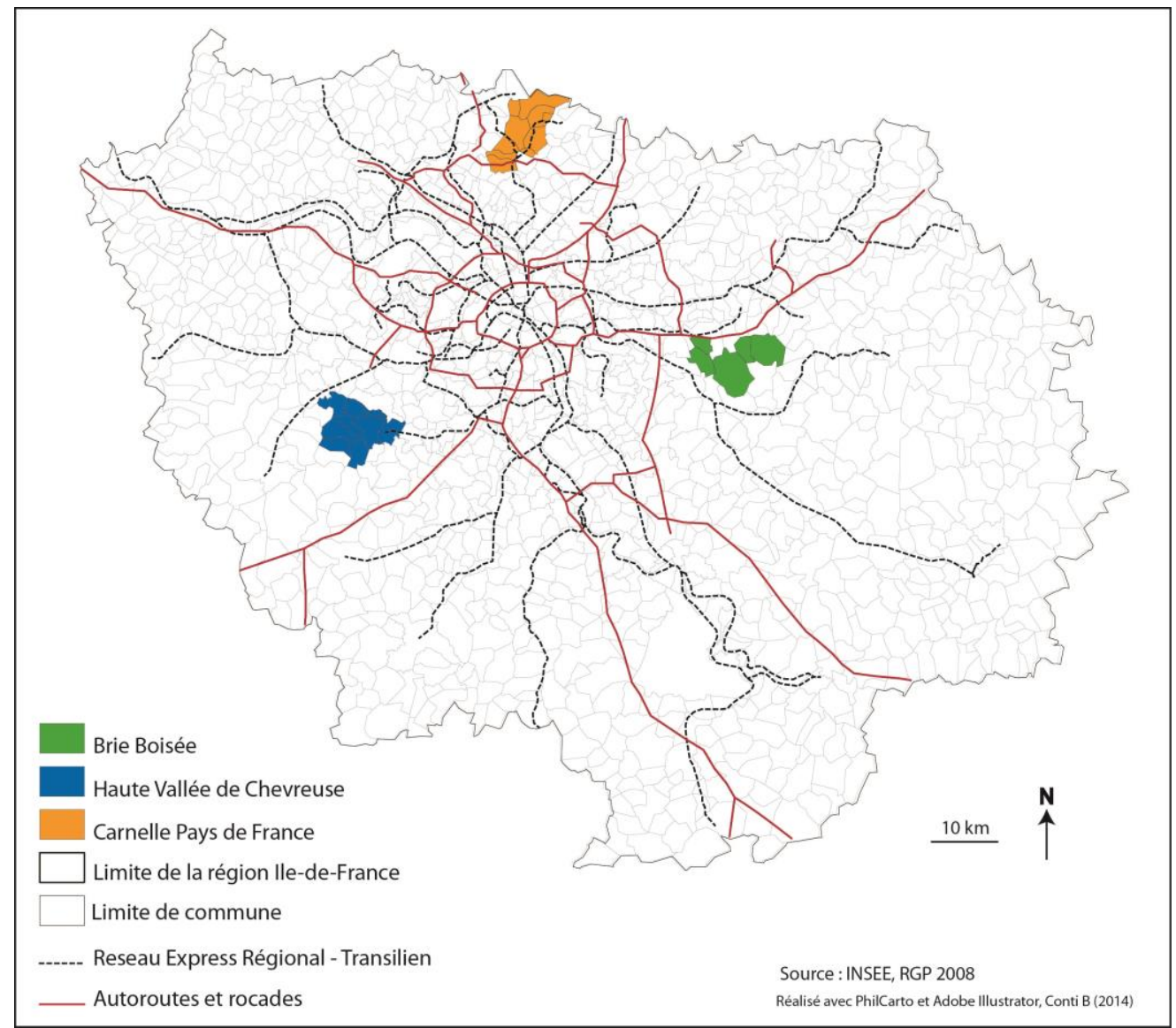

Source : Aguiléra et al., 2014

\section{Les nouvelles dynamiques résidentielles : le vert comme fil rouge}

Les territoires périurbains sont entrés dans une phase plus " mature » (Berger et al., 2014) dont rendent compte d'une part l'hétérogénéité croissante de leur population (les jeunes ménages avec enfants ne sont plus le profil dominant), et d'autre part le renouvellement (et la diversification) des logiques résidentielles : le desserrement depuis Paris ou la proche couronne n'est plus la norme, et une part majoritaire des habitants est issue d'une autre commune périurbaine, généralement située dans le même département. L'implantation périurbaine est, par ailleurs, envisagée sur la durée et non plus comme un choix par défaut. Certes, la cherté de l'immobilier francilien à Paris et en petite couronne est une contrainte importante des choix résidentiels intra-métropolitains. Mais la localisation dans le périurbain procède aussi et surtout d'une dynamique positive liée à une volonté d'amélioration du cadre de vie, en particulier pour les ménages qui habitaient auparavant dans les départements de la petite couronne d'île de France. Dernier argument, les déménagements envisagés sont rares, et répondent principalement à des logiques traditionnelles (changement familial ou professionnel) qui ne remettent en général pas en cause le choix de la localisation périurbaine. 
La proximité avec la nature, en particulier la forêt (beaucoup plus que la possession d'un jardin), constitue une qualité essentielle du cadre de vie. Au-delà de leurs seuls aspects récréatif et sportif (running, vélo, etc.), les aménités naturelles constituent, pour les périurbains, une dimension essentielle de l'identité de leur territoire et de sa singularité au sein de la métropole francilienne. Toutefois les habitants ne se reconnaissent pas dans le concept de périurbain, qui a peu voire pas de sens pour eux, mais font appel à la notion de ruralité : $88 \%$ des personnes ayant répondu à notre questionnaire estiment en effet (et apprécient de) vivre "à la campagne ". Cette revendication de la ruralité, en réalité largement déconnectée de sa dimension agricole, renvoie tout à la fois aux aménités naturelles mais aussi à un mode de vie dont les activités du quotidien se partagent pour l'essentiel entre l'échelle très locale et un territoire d'un rayon d'une quinzaine de kilomètres autour du lieu de résidence. La faible étendue de ce bassin de vie, qui n'est pas associée à un manque d'accessibilité par les habitants enquêtés mais bien au fait de disposer de tout "à proximité ", témoigne aussi d'une forme de maturité des territoires périurbains.

Photo 1:

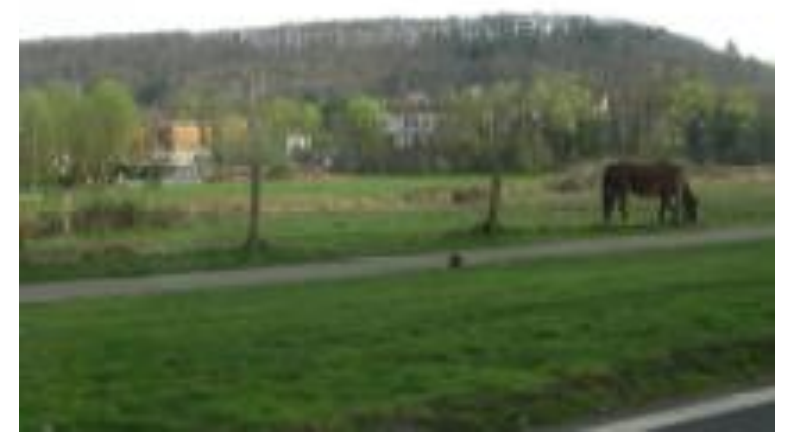

Source : Photo de Vaclav Stransky. (Aguiléra et al., 2014)

\section{La contraction des bassins de vie}

Dès lors qu'elle dispose d'équipements, commerces et services, la commune de résidence tient une place très particulière dans les bassins de vie (Aguiléra et Thébert, 2015). D'après la dernière EGT (2010), près du tiers des déplacements des habitants des trois territoires d'études ont pour destination leur commune. Nos enquêtes montrent de surcroît l'importance mais aussi la variété des ressources qui sont utilisées à l'échelle communale. La commune de résidence n'est ainsi ni un dortoir, ni un simple cadre de vie bucolique. C'est un territoire valorisé et dont la fréquentation (et l'appréciation) augmentent avec le temps.

Les activités liées à la santé (médecin, pharmacie) arrivent en tête des activités qui sont pratiquées de façon préférentielle dans la commune de résidence. La fréquentation des espaces verts n'est, par ailleurs, qu'une dimension des activités récréatives intra-communales, qui incluent aussi des pratiques sportives et culturelles, dont la participation à des événements ponctuels (brocantes, fêtes de village). La visite des amis et de la famille constitue un aspect autre important des activités intra-communales, qui témoigne de l'importance des réseaux amicaux et sociaux de proximité qui ne passent pas seulement par le milieu scolaire mais également par l'implication dans des associations sportives, culturelles, etc., et la participation à des événements (brocantes, fêtes, etc.). Enfin une partie des achats, notamment alimentaires, est effectuée dans la commune de résidence. L'EGT (2010) montre d'ailleurs que 36\% des déplacements pour achat des périurbains de nos territoires sont effectués dans leur commune, et $18 \%$ des répondants à notre enquête par questionnaire citent leur commune de résidence comme premier lieu pour leurs achats alimentaires. 
Photo 2 :

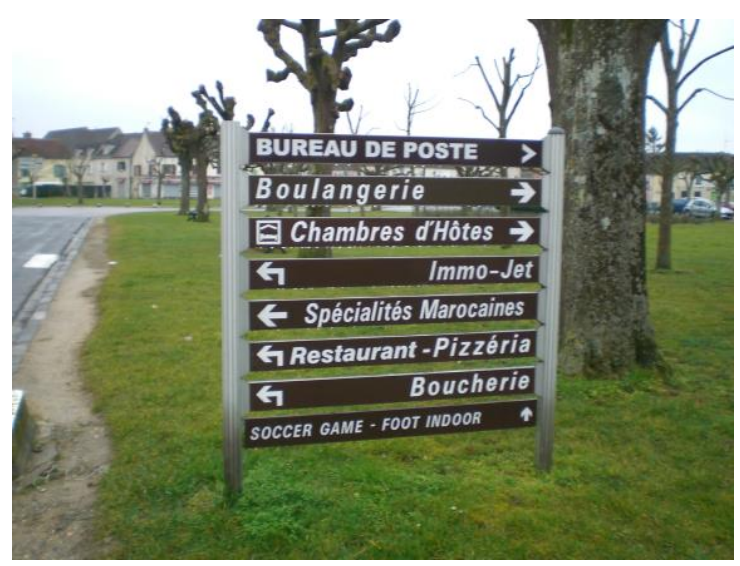

Source : Photo d'Hélène Nessi. (Aguiléra et al., 2014)

Le choix de fréquenter la commune de résidence procède de plusieurs logiques, parmi lesquelles celle du " dépannage » occasionnel n'apparaît que très secondaire. D'une part, la fréquentation des ressources communales est une amorce indispensable au processus d'intégration. D'autre part, elle permet la mise en place d'une relation personnalisée au commerçant ou au producteur qui crée de la valeur ajoutée et différencie ainsi l'offre communale de celle des pôles secondaires, où le consommateur est anonyme. Enfin la consommation locale est aussi un acte « militant » qui soutient le maintien des commerces et services locaux, voire qui alimente pour certains le rejet de l'hyperconsommation et de l'hypermobilité.

La commune de résidence ne constitue plus, malgré tout, le territoire principal du quotidien, contrairement à ce qui caractérise les sociétés rurales " traditionnelles » : plus des trois-quarts des personnes enquêtées par questionnaire déclarent sortir régulièrement de leur commune de résidence en semaine (et un peu plus de la moitié durant le week-end). Tout comme le territoire de l'intercommunalité, qui n'a que peu de sens au plan des activités quotidiennes: les trois-quarts des déplacements qui ont lieu à l'intérieur des communautés de communes concernent en réalité la seule commune de résidence.

En dehors de leur commune et de leur intercommunalité, les activités des périurbains sont principalement structurées par quelques grands pôles secondaires du périurbain francilien : villes nouvelles, centres commerciaux, communes d'une certaine taille.. Ces pôles secondaires, dont le développement au cours des dernières décennies repose sur des dynamiques à la fois locales (liées à la périurbanisation des ménages, qui a fait augmenter la demande) et métropolitaines (desserrement des emplois et installation de centres commerciaux d'ampleur régionale sur des terrains vastes et peu chers, en bordure d'autoroutes), limitent les distances parcourues et ont même permis une contraction des bassins de vie au cours des dernières décennies, y compris pour les motifs professionnels (Figure 2). 
Figure 2 : Évolution de la portée moyenne (en km) des déplacements selon le motif entre 1983 et 2010 (hors marche à pied)

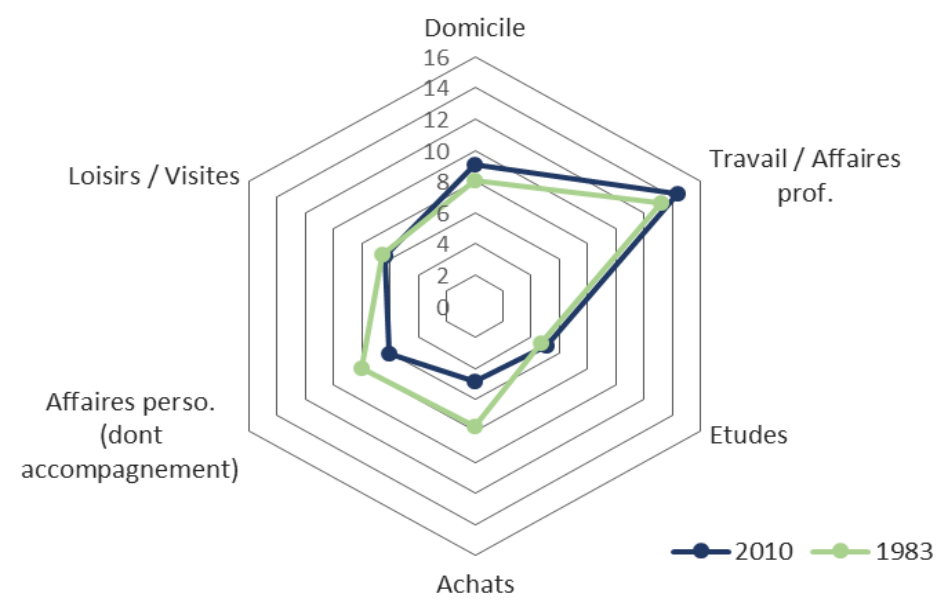

Source: EGT 2010-2011 STIF-OMNIL-DRIEA; EGT 1983 DRIEA-INSEE. Calculs des auteurs (L. Proulhac ?)

Le desserrement multi-polarisé des emplois franciliens n'a pas permis de réduire de façon significative les distances domicile-travail, mais en a limité l'allongement: après une période de croissance importante jusqu'au recensement de 1990, due à l'effondrement du modèle antérieur où l'emploi (principalement agricole) était essentiellement localisé dans la commune de résidence, la distance moyenne domicile-travail (calculée à vol d’oiseau) a stagné, et marque même un léger fléchissement sur la période 1999-2008 (Figure 3). La baisse, comme illustré dans la Figure 3, de la dispersion des distances domicile-travail, montre bien ce passage d'un système de mobilité dual, où une partie de la population était dépendante de Paris et l'autre travaillait très localement (dispersion forte en 1968 et encore en 1975) à un système plus homogène où la distance moyenne est plus forte (double en 2008 par rapport à 1968) mais où les déplacements les plus longs et les plus cours se sont tassés au profit d'une forte croissance des déplacements de portée médiane, en partie vers les pôles périphérique (Figure 4).

Figure 3 : Distance domicile-travail des actifs résidant dans les trois intercommunalités. 


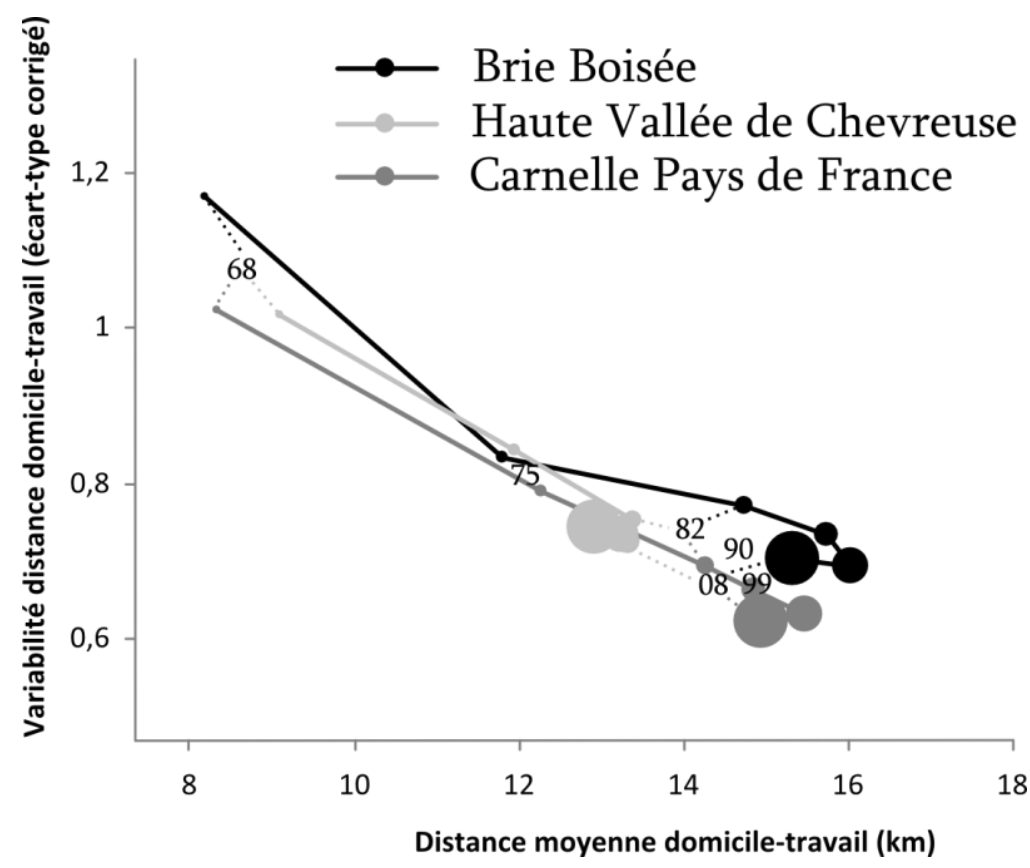

Source: B. Conti (2012), recensement de la population, INSEE. L'axe des ordonnées montre l'écarttype de la distribution des distances, corrigé de l'effet de croissance "statistique " lié à l'asymétrie de la distribution des distances domicile-travail. L'écart-type corrigé est l'écart-type de la distribution lognormale collant le mieux à la distribution des distances observée à chaque date, pour chaque territoire.

Cette réorganisation des bassins de vie en faveur de la proximité implique que le périurbain francilien est devenu de moins en moins dépendant du cœur de la métropole, y compris en matière d'emplois (Figure 4). Ainsi, le temps de trajet moyen domicile-travail se situe désormais dans les territoires étudiés autour de 40 minutes, moyenne, ce qui cache encore de réels contrastes : la moitié des personnes enquêtées habitent en effet à moins de 30 minutes de leur lieu de travail, tandis que seulement un peu moins d'un-tiers ont plus d'une heure de trajet (il s'agit notamment de ceux qui travaillent à Paris).

Figure 4 : Lieu d'emploi des actifs résidant de la communauté de commune de la Brie Boisée.

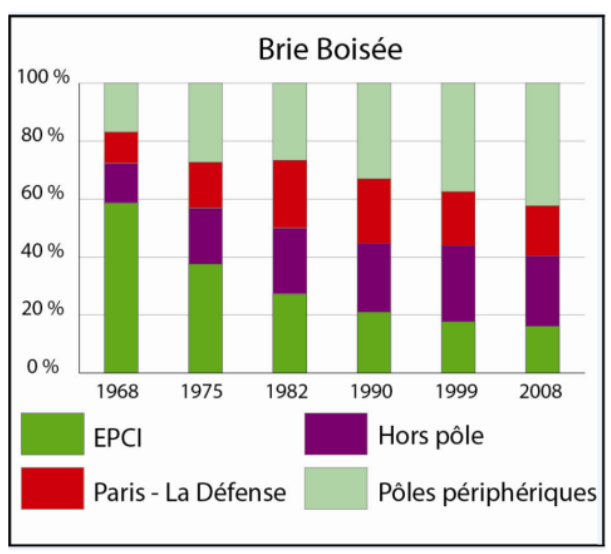


Source : B. Conti (2012), recensement de la population, INSEE.

Outre le desserrement des activités, commerces et services, les raisons sont aussi à rechercher du côté des contraintes de transport inhérentes à une grande métropole. Soit Paris est un lieu d'emploi, auquel cas il est une destination régulière, soit il ne l'est pas et alors il n'est fréquenté que de façon ponctuelle et sélective, pour accéder à certaines aménités très spécifiques dans le domaine de la santé et de la culture en particulier. En revanche les communes des départements de la petite couronne sont très peu fréquentées, hormis dans le cadre professionnel.

\section{Vers des mobilités périurbaines plus durables? Quelques pistes pour l'action} publique

Malgré une tendance à la contraction des bassins de vie, le bilan environnemental des mobilités périurbaines n'en est pas pour autant vertueux.. Parce qu'ils parcourent des distances plus élevées et qu'ils utilisent davantage l'automobile, les habitants (et tout particulièrement ceux qui font partie des aires urbaines de plus de 500000 habitants) émettent pour leurs déplacements quotidiens significativement plus de gaz à effet de serre que les urbains (Longuar et al., 2010). Et le taux de motorisation des ménages périurbains a continué à progresser depuis le milieu des années 90 , alors qu'il est en stagnation dans l'urbain, et même en recul dans Paris (Robin, 2010).

La voiture reste l'outil indispensable du mode de vie périurbain. Elle est d'ailleurs autant une cause qu'une conséquence de l'agencement dans l'espace et dans le temps des programmes quotidiens. Elle permet de "faire tenir" dans un temps contraint des activités qui peuvent impliquer les échelles locale et métropolitaine au cours d'une même journée. Elle est aussi garante d'une grande flexibilité des programmes d'activités : nos enquêtes montrent clairement un entre usage de l'automobile et faible programmation des activités non contraintes. Cette recherche de flexibilité est aussi un frein au covoiturage, qui est peu pratiqué hormis pour les activités péri-scolaires ou lorsqu'il s'agit de rendre service à des personnes âgées non motorisées.

Le faible recours aux transports en commun repose sur des facteurs explicatifs bien connus. II tient à l'inadaptation de l'offre, principalement conçue pour le rabattement sur les axes lourds qui desservent la capitale (notamment le RER), sur des créneaux horaires qui sont essentiellement ceux des heures de pointe du matin et du soir. L'accès aux pôles secondaires, y compris pour motif professionnel, est souvent compliqué, en particulier pour les emplois situés dans les zones d'activité éloignées des grands réseaux de transport public, et mal desservis en interne par les rares bus qui y accèdent. Par ailleurs, si la contraction des bassins de vie a permis de modérer les distances parcourues, elle a plutôt favorisé l'usage de l'automobile en diminuant les liens avec Paris, qui sont ceux pour lesquels la part des transports en commun est la plus élevée, au profit des pôles secondaires. Seul le recours croissant à la commune de résidence déroge à la règle, les faibles distances en jeu étant favorable à la marche à pied (mais peu le vélo, qui reste surtout utilisé pour les promenades ou pour un usage sportif).

La situation semble donc compliquée, avec d'un côté des habitants qui aspirent à vivre dans des territoires périurbains et des territoires qui " s'organisent " au fil des années pour répondre à cette demande, et de l'autre côté l'impératif de réduire considérablement les émissions de gaz à effet de serre causés par leurs déplacements, qui met la périurbanisation et les périurbains au banc des principaux accusés de l'augmentation des émissions. Mais cette recherche montre aussi un certain nombre de signes encourageants, que des politiques de transport et d'aménagement adaptées pourraient encourager afin de favoriser le développement de pratiques plus durables. 
La première "bonne nouvelle » est que les périurbains sont en demande de " proximité ». Proximité qui, par ailleurs, va au-delà de la simple logique de minimisation des distances : on l'a vu, la fréquentation de la commune de résidence est aussi de nature sociale et identitaire. La seconde " bonne nouvelle " est que les habitants montrent des signes de lassitude vis-à-vis du modèle tout-automobile. La voiture est un mal nécessaire au déploiement d'un mode de vie qui lui est, en général, très apprécié. Les reproches faits à l'automobile par les ménages concernent principalement non pas son coût financier (qui, on le sait, est souvent sous-évalué par les ménages) ni (encore moins) son impact environnemental, mais plutôt la perte de temps associée. C'est tout particulièrement le cas pour ceux qui subissent quotidiennement la congestion sur leur trajet domicile-travail aux heures de pointe, ou encore ceux qui jouent les "taxis " pour emmener leurs enfants aux activités péri-scolaires. La troisième "bonne nouvelle » est que les ménages périurbains considèrent qu'ils disposent d'un certain nombre de marges de manœuvre pour réduire leur usage de la voiture, sans (trop) altérer leur mode de vie ni remettre en question leur choix résidentiel. Moins du quart des personnes ayant répondu à notre questionnaire estiment qu'elles ne peuvent (ou ne veulent) rien changer à leur mobilité (Tableau 1). Le renoncement complet à la voiture n'est pas à l'ordre du jour : il est de toute façon trop compliqué à mettre en œuvre, en l'état actuel des formes urbaines et des modes de vie. Mais nos enquêtes suggèrent que les habitants sont prêts à basculer vers des formes de transport collectives pour certains trajets, et à réduire les kilomètres parcourus si les formes urbaines le leur permettent, ou en cas de forte augmentation des carburants. A ce sujet, nos analyses, comme d'ailleurs celles menées à peu près à la même période par X. Desjardins et L. Mettetal (2013), montrent toutefois qu'une hausse, même sensible, des prix à la pompe n'aura, au moins à moyen terme, qu'un effet limité sur l'usage de la voiture, les ménages étant prêts à des sacrifices importants, y compris financiers, pour continuer à utiliser l'automobile (et à habiter le périurbain).

Le simple fait que la réponse majoritaire au questionnaire sur les potentiels de réduction de l'usage de l'automobile soit deux modalités d'offre plus adaptée aux pratiques automobiles des ménages montre que ceux-ci mettent la personnalisation et la flexibilité au cœur de leurs souhaits de mobilité. Pour les pouvoirs publics, un enjeu majeur se dessine autour de la consolidation de ces bassins de vie de proximité, dans une perspective de réduction des distances parcourues et, au moins pour certains flux, de report modal vers les modes doux. Au plan social, le bénéfice potentiel est un renforcement des capacités d'adaptation du périurbain à une crise énergétique sévère. De façon concrète, il est utile de distinguer les motifs professionnels des autres, parce qu'ils ne se déploient pas sur les mêmes territoires et ne concernent ni les mêmes horaires, ni les mêmes jours de la semaine. Pour l'emploi, des enjeux forts se dessinent autour de la consolidation des pôles secondaires et de leur desserte par des services très souples de transport collectif et/ou, selon les cas, de véhicules partagés. Les ménages expriment d'ailleurs des attentes à l'égard de tels services de mobilité (environ 15\% des ménages), mieux adaptés à leurs horaires et à la géographie de leurs trajets (Tableau 1). Les réseaux sociaux constitués localement sont plutôt un support favorable au développement de ces pratiques.

Tableau 1 : Potentiel perçu de réduction de l'usage automobile

\begin{tabular}{|l|c|c|c|c|}
\hline $\begin{array}{l}\text { Qu'est-ce qui pourrait vous inciter } \\
\text { à utiliser moins votre véhicule ? } \\
\text { (plusieurs réponses possibles) ॥ }\end{array}$ & Ensemble & Brie boisée & $\begin{array}{c}\text { Carnelle Pays de } \\
\text { France }\end{array}$ & $\begin{array}{c}\text { Vallée de } \\
\text { Chevreuse }\end{array}$ \\
\hline $\begin{array}{l}\text { Transports en commun mieux } \\
\text { adaptés à nos trajets }\end{array}$ & $53,3 \%$ & $52,4 \%$ & $53,9 \%$ & $52,9 \%$ \\
\hline $\begin{array}{l}\text { Transports en commun mieux } \\
\text { adaptés à nos horaires }\end{array}$ & $41,8 \%$ & $41,0 \%$ & $45,2 \%$ & $37,9 \%$ \\
\hline $\begin{array}{l}\text { Possibilité de travailler certains } \\
\text { jours à domicile }\end{array}$ & $26,8 \%$ & $28,6 \%$ & $21,7 \%$ & $31,0 \%$ \\
\hline Forte hausse des carburants & $26,7 \%$ & $27,6 \%$ & $25,2 \%$ & $27,6 \%$ \\
\hline
\end{tabular}




\begin{tabular}{|l|c|c|c|c|}
\hline $\begin{array}{l}\text { Plus de commerces et services dans } \\
\text { la commune }\end{array}$ & $23,9 \%$ & $14,3 \%$ & $31,3 \%$ & $26,4 \%$ \\
\hline $\begin{array}{l}\text { Vous ne souhaitez ou ne pouvez } \\
\text { utiliser moins votre véhicule }\end{array}$ & $23,2 \%$ & $24,8 \%$ & $23,5 \%$ & $20,7 \%$ \\
\hline $\begin{array}{l}\text { Itinéraires vélo sécurisés vers les } \\
\text { communes des environs }\end{array}$ & $16,3 \%$ & $19,0 \%$ & $13,0 \%$ & $17,2 \%$ \\
\hline $\begin{array}{l}\text { Accès à un service de transport à la } \\
\text { demande }\end{array}$ & $13,4 \%$ & $8,6 \%$ & $16,5 \%$ & $14,9 \%$ \\
\hline $\begin{array}{l}\text { Amélioration des cheminements } \\
\text { pour les piétons }\end{array}$ & $8,5 \%$ & $10,5 \%$ & $7,0 \%$ & $8,0 \%$ \\
\hline
\end{tabular}

Source : enquête par questionnaire réalisée en 2013 auprès de 300 ménages périurbains.

Pour les activités non professionnelles, les enjeux se situent à la fois à l'échelle des pôles secondaires mais également à une échelle plus locale, celle des communes et aussi des inter-communalités, dont les potentialités sont pour l'instant sous-exploitées. Il s'agit, bien sûr, d'étoffer l'offre de commerces et services. Mais l'enjeu est aussi et surtout de mieux hiérarchiser et articuler leur structure spatiale à l'intérieur des bassins de vie. La tâche est complexe, car on se heurte rapidement à la fois à des problèmes de périmètres institutionnels (en particulier, le territoire dessiné par les pôles secondaires n'a pas de réalité politique) et économiques: le développement largement anarchique des centres commerciaux est source de recettes fiscales, et la promesse (du reste pas toujours vérifiée) de création d'emplois locaux constitue un argument politique puissant. Outre l'objectif d'une meilleure organisation spatiale des mobilités pour les achats et les loisirs, qui favorise une réduction des distances, celui d'un report modal doit également être poursuivi, même s'il apparaît plus compliqué eu égard à la dispersion spatiale et temporelle des déplacements en jeu.

À l'échelle intra-communale, il faut encourager encore plus le recours à la marche et au vélo par des actions sur les trottoirs, les pistes cyclables, les cheminements piétons, notamment sur les trajets à destination des établissements scolaires (voire des activités péri-scolaires).. Le développement des mobilités intercommunales et leur basculement vers des modes doux, notamment le vélo, constitue un autre défi. Une réflexion concernant une meilleure distribution des commerces et services à cette échelle est à cet égard intéressante. Cela permettrait de favoriser les déplacements de courte distance tout en résolvant potentiellement certains problèmes, comme la viabilité économique des activités qui s'installent dans les petites communes périurbaines .ou de réduire les réticences exprimées par les populations de voir leur commune accueillir un développement important qui aurait pour effet de faire (trop) augmenter la population. En effet, l'attachement à la ruralité est associé à une volonté de préservation du caractère villageois de la commune (Charmes, 2007).

Dans un autre registre, qui concerne moins directement les acteurs locaux, nos enquêtes montrent que développer les possibilités de télétravailler certains jours à domicile constitue pour certaines professions (notamment les cadres) une perspective intéressante pour réduire l'usage de la voiture, en particulier si les carburants augmentent fortement.

\section{Conclusion}

Le mode de vie périurbain, décrypté par l'analyse des mobilités quotidiennes, s'est profondément transformé au cours des dernières décennies sous l'effet de la croissance démographique et du développement de commerces, de services à la population et aussi d'emplois. Malgré tout, la dépendance à l'automobile reste forte.

Si les pratiques alternatives de mobilité (comme le covoiturage) sont très marginales, le chaînage des déplacements plutôt limité, l'usage des transports en commun réservés aux scolaires et aux trajets domiciletravail vers Paris, et le recours aux modes non mécanisés (vélo, marche à pied) cantonnés aux trajets intracommunaux ou aux loisirs, les périurbains franciliens semblent montrer de réelles aptitudes au changement 
vers des mobilités moins fondées sur l'automobile. Les évolutions ne semblent pas être seulement du ressort du discours, mais s'incarnent dans des solutions concrètes qui, au premier chef, concernent une rationalisation de l'usage de la voiture, ou le passage à des modèles hybrides voire électriques (si les prix diminuent). Toutefois des analyses plus approfondies montrent que les capacités d'adaptation sont plus vastes, et qu'elles elles reposent plus sur des réaménagements des programmes d'activité (lieux de consommation et chaînage des activités) que sur l'usage des TIC comme substitut à la mobilité physique.

La mise en œuvre de changements conséquents dans les pratiques de mobilité doit s'appuyer sur les dynamiques en cours, exploiter en quelque sorte la " bonne volonté » des habitants et les faiblesses de plus en plus évidentes à leurs yeux d'un système basé sur le tout automobile. Les périurbains ne sont pas réfractaires à l'idée de changements, dès lors qu'ils leur permettent de demeurer dans le périurbain, de continuer à déployer leurs activités entre l'échelle locale et métropolitaine (où sont notamment situés les emplois), voire qu'ils améliorent leur quotidien, en diminuant la pénibilité associée à certains usages de la voiture qui occasionnent trop de perte de temps (alors que d'autres usages, fondés sur caractère flexible de ce mode de transport, sont au contraire valorisés).

Les habitants ne sont pas particulièrement réfractaires à l'idée de devoir moins utiliser leur voiture, voire de se démotoriser partiellement (d'autant plus que le gain financier serait important). Toutefois en l'état actuel des modes de vie, des systèmes de transport et des formes urbaines on ne peut pas compter uniquement sur des changements de pratiques individuelles ou sur une hausse même forte des carburants, qui pénaliserait surtout les plus modestes et les habitants des communes les moins bien dotées en commerces et services, ou en services de transports collectifs. Par ailleurs la progressive démocratisation des véhicules hybrides et électriques, de même que les rapides progrès des véhicules thermiques en matière de consommation de carburant, rendent fragiles des politiques qui compteraient principalement sur la hausse des prix à la pompe pour réguler la mobilité périurbaine (voire la périurbanisation).

Pour favoriser des changements significatifs, des politiques articulant aménagement et transport sont donc indispensables. Elles doivent s'appuyer sur (et conforter) les tendances actuelles de développement des équipements, commerces et services à proximité des lieux de résidence, tout en reconsidérant la cohérence de ces nouvelles formes urbaines à l'échelle des bassins de vie des périurbains. L'articulation avec les offres de transport doit également être repensée, en prenant en compte à la fois les transports collectifs, la marche à pied et le vélo, mais également les nouveaux services de mobilité (covoiturage, auto-stop de proximité, etc.), là aussi à différentes échelles spatiales. Les difficultés sont importantes. Elles relèvent notamment de la multiplicité des périmètres institutionnels en matière de transport et d'aménagement, mais aussi de la volonté des habitants de contenir le développement de leur commune de résidence.

D'autres défis attendent les territoires périurbains. En matière de mobilité, les enjeux ne concernent pas seulement les pratiques de mobilité des habitants, mais également celle des non résidents qui viennent de façon croissante travailler (en semaine) mais aussi faire leurs achats et se divertir (le week-end), et qui viennent principalement en voiture (Aguiléra et Voisin, 2014). En particulier, les emplois qui se développent dans les trois territoires étudiés ici sont de plus en plus occupés par des actifs qui habitent en dehors du périmètre communal (Conti, 2012). Au plan économique, les emplois offerts par le périurbain, y compris les pôles secondaires, demeurent faiblement diversifiés et ils ont par ailleurs été moins dynamiques sur la dernière décennie que dans la partie centrale (Paris et petite couronne) de la métropole (Terral et al., 2015). En matière d'aménagement, les nouvelles gares et lignes de métro du Grand Paris peuvent rapidement bouleverser assez profondément les stratégies résidentielles sur nos territoires (avec des risques de "gentrification 》), en particulier la forme des bassins de vie de leurs habitants, selon des modalités qui restent largement inconnues. Enfin le développement de véhicules peu gourmands en énergie (véhicules électriques et hybrides, ou véhicules thermiques consommant 2 litres aux $100 \mathrm{~km}$ ) pourraient, s'ils se démocratisent, figer le système actuel construit autour d'un usage quasi-exclusif et principalement individuel de l'automobile. 


\section{Bibliographie}

Aguilera A., Bonin O., Conti B., Coralli M., Le Néchet F., Nessi H., Proulhac L., Sajous P., Stransky V., Terral L., Thébert M. (2014). «Dynamique du peuplement, des formes urbaines et des mobilités dans les territoires de la périurbanisation. Quels enseignements au regard des enjeux du développement durable ? ", Rapport PUCA, p. 264

Aguiléra A., Thébert M. (2015). La place de la commune de résidence dans les modes de vie périurbains. Colloque « Construire les proximités dans un monde global », Tours, 20-22 mai.

Aguiléra A., Voisin M. (2014). Urban form, commuting patterns and CO 2 emissions: What differences between the municipality's residents and its jobs?. Transportation Research Part A: Policy and Practice, 69, 243251.

Bailly A., Bourdeau-Lepage L. (2011). Concilier désir de nature et préservation de l'environnement: vers une urbanisation durable en France. Géographie, économie, société, 13(1), 27-43.

Billard G., Brennetot A. (2009). Le périurbain a-t-il mauvaise presse? Analyse géoéthique du discours médiatique à propos de l'espace périurbain en France. Articulo-Journal of Urban Research, (5).

Charmes E. (2007). Les Périurbains sont-ils anti-urbains? In Les Annales de la recherche urbaine (No. 102, pp. 7-18).

Conti B, (2012). Dynamique de peuplement et de mobilité du périurbain francilien. Quels enseignements au regard de la durabilité urbaine ?, Mémoire Master 2, Université Paris-Est.

Desjardins X., Mettetal L. (2013). L'habiter périurbain face à l'enjeu énergétique. Flux, 89(3), 46-57.

Feildel B., Bailleul H., Laffont G-H. (2014). Les imaginaires de la mobilité. De possibles ressorts pour la mise en durabilité des espaces périurbains ?. Recherche Transports Sécurité, NecPlus, 2014, Les sens des circulations, 30 (2-3), 143-160.

Longuar Z., Nicolas J.-P., Verry D. (2010). Chaque Français émet en moyenne deux tonnes de CO2 par an pour effectuer ses déplacements. La Revue du CGDD, 163-176.

Robin M. (2010). La motorisation des ménages continue de s'accroître au prix d'un vieillissement du parc automobile. La Revue du CGDD, 99-121.

Terral L., Aguiléra A. (2015). "La périurbanisation de l'activité économique dans l'aire urbaine parisienne. Le desserrement de l'emploi joue-t-il un rôle ? ", 52ème colloque de l'Association de Science Régionale de Langue Française (ASRDLF), Territoires méditerranéens : agriculture, alimentation et villes, Montpellier, France, 7-9 juillet.

Vanier M., Roux E. (2008). La périurbanisation : problématiques et perspectives. La documentation française. 